\title{
From artificial societies to new social science theory
}

\author{
Eric Silverman and John Bryden \\ School of Computing, University of Leeds, Leeds LS2 9JT \\ erics: johnb@comp.leeds.ac.uk
}

\begin{abstract}
We identify two distinct themes in social science modelling. One, more specific, approach is that of social simulation which addresses how behaviour of many actors can lead to emergent effects. We argue that this approach, while useful as a tool in social science policy development, is fundamentally constrained due to the fact that its models are developed within the society they are supposed to model. Alternatively, the second theme looks to take a more holistic view by taking inspiration from systems sociology. This approach looks to build societies from the bottom up and may allow us to generate new perspectives in social theory.
\end{abstract}

\section{Introduction}

Understanding and predicting the future of human society is a problem of staggering complexity. Our level of understanding of the basic unit of society, the human being, is far from complete, let alone the collective interactions and transmissions of information between the 6 billion individuals that live on our planet. The mind boggles over the potentials for chaotic effects and intractable nonlinearities in this vast system.

That said, the problem of how life has evolved on that same planet would seem also to be one of insurmountable complexity if it weren't for the theories of Charles Darwin [1]. Perhaps there is hope for a theory in social science of similar explanatory power to evolution: one that does not fully explain society, but provides us with a holistic framework to push forward our understanding of society - in a similar way that evolution does for biology. This is the problem at hand, the development of a fundamental theory of social science.

The only sensible way we may approach such a problem is by using the best tools available in the most appropriate manner. We propose that computer modelling gives us the freedom of abstraction and the power of the study of non-linear mechanisms that will be vital for this endeavour.

This paper looks at the current literature surrounding social simulation to attempt to formulate the role computer simulation should play in working towards fundamental theories of social science. From our review, we outline and distinguish two main themes in the computer modelling of social systems: social simulation and systems sociology. One key common feature of both these modelling themes is that both focus on producing emergent macro-level effects. 
The distinction we develop between social simulation and systems sociology is based on the application and approach of these two themes. The application of social simulation models is through the guidance of social policy. Such models are often linked, or intended to be linked, to empirical data. As such social simulation models are bottom up in their application. Systems sociology models however are top down in their application - they are intended to push forward social science and its paradigms.

When we consider the approach of the two themes, they take the opposite roles to those taken in their application. Social simulation models take a top down approach on human behaviour, identifying a specific class of behaviour and predicting macro-level patterns. We argue that it is the very specificity of this approach that restricts its usefulness in creating a new social theory. In contrast, systems sociology modelling takes a bottom up view, starting with building blocks of social behaviour and attempts to form meaningful and recognisable social structure. Unlike the social simulation approach, the building blocks are not defined with any predetermined interactions.

In many ways this looks analogous to the perspectives of evolutionary biology and artificial life - with evolutionary biology concerning itself with microevolution in specific biological systems and artificial life concerning itself with macroevolutionary theory from a more general perspective. However, evolutionary theory was founded on a generalisation from observances from specific systems from the Galapagos islands [1]. We argue that specific social systems are not likely to generalise into a fundamental theory for social science in this way. Being human beings, and therefore part of the system being modelled, we are not able to look at our society from the outside in the way we may look at nature. We propose that we require the input from grown artificial societies to give us new perspectives on our own society.

The next section introduces key literature in the field of social simulation and criticises social simulation from the perspective outlined in this Introduction. Following on from this a new perspective derived from systems sociology is introduced. Finally the two themes are compared in more detail before the paper is concluded.

\section{Social simulation}

This section on the social simulation stream focuses mainly on the work of two seminal social science modellers - Schelling and Cederman. It starts by critiquing a famous case study: Schelling's model of residential segregation and its impact upon the development of social simulation. Following this, we consider a broader picture of the stream looking at Cederman's three types of social simulations. Finally, we look at some of the relevant criticisms of social simulation seen in the current literature. 


\subsection{Case study: Schelling's segregation model}

Schelling's well-known 'chequerboard model' of residential housing segregation has proves an interesting example of the power of the social simulation theme in certain aspects of the social sciences[2]. He sought to confirm his suspicion that if the racial makeup of a given area was critical to an individual's choice of housing, then even populations of individuals tolerant to mixed-race environments would end up segregating into single-race neighbourhoods. To this end, he devised a simplistic model of two populations placed on a chequerboard, with each agent on the board given a very simple rule: if the number of agents of a different 'race' in its immediate eight-cell neighbourhood exceeded a certain critical threshold, the agent would become 'unhappy' and move; otherwise, the agent would remain in its current position.

Schelling's model supported his initial hypothesis, proving that even if the agents were given a high level of tolerance for other agents of a different 'race,' the agents would end up segregating themselves into separate neighbourhoods. The model also illustrated a deeper point: large-scale factors such as socio-economic or educational differences between different ethnic populations could not explain the generally puzzling phenomenon of residential segregation. Indeed, without a greater insight into the preferences and thought processes of individuals within a given population (their 'micromotives'), some critical aspects of this social problem may elude the researcher.

Despite its apparent simplicity, Schelling's model provides an important illustration of the potential power of the social simulation theme. The chequerboard model provides only the barest of motivations for each agent in the simulation, and yet the results are very applicable to more complex theories of the residential segregation phenomenon. As he states, a model 'can be a precise and economical statement of a set of relationships that are sufficient to produce the phenomena in question, or, a model can be an actual biological, mechanical, or social system that embodies the relationships in an especially transparent way, producing the phenomena as an obvious consequence of these relationships' [3] [p. 87]. Schelling's model shows the power of this transparency, demonstrating a clear relationship between the simple micromotives driving an agent's movement between neighbourhoods and the resulting segregation.

Perhaps most importantly, Schelling's model succeeds due to its obvious qualitative similarity to the problem of residential segregation: one can see the effect of varying levels of tolerance in these agents by simple visual examination, as the agents begin to cluster into separate neighbourhoods of their own accord. While Cederman and many others acknowledge that social simulation models can be difficult to analyse, models of this type which illustrate important social concepts simply could in fact be much easier to effectively 'analyse' through these qualitative similarities than more detailed models which aim for social realism. In fact one might argue that such a perspective echoes the techniques of much of social science.

While Schelling's model was accepted as a remarkable illustration of a simple principle regarding the large-scale effects of individual housing preferences, 
some modellers sought to create a more 'realistic' Schelling-type model which could incorporate socio-economic factors as well[4-6]. Given the accepted complexity of residential segregation as a social problem, and the new insight into the effects of individual preference illuminated by Schelling, models incorporating both Schelling-style 'micromotives' and large-scale social factors were seen as a potential method for examining the interactions between these two levels of social structure, something that was very much lacking in Schelling's original formulation.

However, even increasing the complexity of Schelling's rather elegant portrayal of this particular societal problem cannot solve a fundamental problem of this approach. Human society by its very nature is a complex system, incorporating as it does the collective interactions and transmission of knowledge of many millions of individual actors in concert. Those interactions and retained societal knowledge can in turn influence members of the society at large, and as a society continues to evolve its past history can exert a tremendous influence on its collective future decisions, making human society heavily path-dependent (see[7] for one example of this phenomenon).

With this in mind, while agent-based models following Schelling's example can provide remarkable insight into the emergence of social phenomena within a certain limited scope, models of this type are inherently limited in their theoretical application by their specificity. As we move into our discussion of Cederman's contribution to social simulation, we will examine these potential methodological difficulties in greater detail.

\subsection{Cederman's three types of social simulation}

Lars-Erik Cederman has been one of the most prominent proponents of agentbased modelling within the political sciences. His own work appears to have substantial roots in the social simulation mould described above, with a more detailed focus on specific aspects of modern political structures [8]. Far from confining himself to a singular mode of enquiry, however, Cederman has developed a theoretical framework of three types of social models in an attempt to outline how a number of potential methods might contribute to the development of social science as a whole [9].

Type 1 models in Cederman's framework focus on behavioural aspects from human society. By implementing a behavioural aspect at the micro-level in the model, macro-level, or emergent, conclusions can be drawn. For example, Axelrod's work has aimed to show the emergence of cooperation, and by using the iterated prisoner's dilemma showed that cooperation is possible in social settings as long as the interactions of the involved agents are iterated and that agents are capable of remembering previous actions [10].

Cederman identifies Type 2 models as those which attempt to explain the emergence of particular configurations in a model due to properties of the agents involved[9]. Cederman's later simulations, for example, focused on issues such as nationalist insurgency and state formation and stability using a framework in which agents within the simulation were assigned specific properties that 
influenced the resultant distribution of resources and information [11,12]. Ian Lustick's Agent-Based Identity Repertoire Model (ABIR) in which agents were provided with potential cultural 'identities' similarly aims to demonstrate the emergence of clusters of these identities through properties of the agents and the changes resulting from their local interactions [13].

Finally, Cederman identifies Type 3 models as being perhaps the most ambitious: these models attempt to model both the agents themselves and their interaction networks as emergent features of the simulation[9]. He cites the field of artificial life as one likely to inform this area of computational work in political science, given that ALife focuses on such emergent features. He also acknowledges that some overlap can occur these three types of models; for example, a Type 1 model may stray into Type 3 territory by allowing agents more latitude in choosing interaction partners, thus allowing for an interaction network to emerge from the system. He argues that such models may provide very powerful tools for the political scientist, allowing for potentially profound conclusions to be drawn regarding the development of political institutions. This approach does seem most methodologically difficult, however, as the already significant abstractions necessary to create Type 1 or Type 2 models must be relaxed even further to allow for such ambitious examinations of emergent features in social systems.

\subsection{Criticisms of social simulation}

From our perspective of attempting to develop a social theory, we present some criticisms of social simulation. These help to elucidate the path we expect social simulation to take in the future. We can then put that into context within our main distinction.

Criticism of agent-based models in political science has come from a number of different areas, but many of those criticisms focus on the difficulty of making sensible abstractions for social and political structures within such a model. One can view potentially all of scientific enquiry as reflecting the inherent biases of the experimenter, and this problem of inherent theory-dependence is even more acute when constructing simulation models [14]. While even abstract ALife models may reference the real-life behaviour of natural biological systems, and the wealth of related empirical data, political models do not necessarily have that luxury. Highly complex social structures and situations, such as Cederman's models of nationalist insurgency and civil war $[11,12]$ involve further layers of abstraction, often involving factors which do not immediately lend themselves to quantification, such as cultural and national identities.

In addition, sociological theory is notoriously difficult to formalise, incorporating as it does a number of both higher- and lower-level cognitive and behavioural interactions. In fact, sociological theory may not benefit from the micro/macro distinction of levels of analysis that benefits researchers of evolution and other large-scale processes [15]. These interacting social levels cannot be clearly differentiated into a hierarchy of processes, making simulation a very difficult, and highly theory-dependent, exercise. 
Of course, with such complex phenomena contributing to the results of a social simulation, finding an appropriate method of analysing those results becomes a very real concern for the modeller. Donald Sylvan's review of Cederman's Emergent Actors demonstrates this common complaint levelled at agent-based models in the social sciences; he urges readers to note carefully the lack of data in its conventional sense throughout Cederman's work [16].

As Sylvan points out, Cederman's 'data' only relate to the interactions of virtual states in an idealised grid-world; applying such data to real-life political events or transitions seems suspect at best. The levels of complexity at work in large-scale political events may be very difficult to capture in an agent-based model, and knowing when to draw a specific conclusion from a model of such an inherently difficult-to-analyse situation is a thorny issue.

While there is a potential for social simulations to illuminate the emergence of certain properties of political structures, the difficulty in connecting these abstracted simulations to real-world political systems is significant. Weidmann and Gerardin[17], with their GROWLab simulation toolkit, have attempted to sidestep these concerns by making their framework compatible with GIS (geographic information system) data, in order to allow 'calibration with empirical facts to reach an appropriate level of realism.'

Putting this into context, the need for an empirical perspective inherently restricts the scope of social simulation models. While such a perspective allows for models to have potential relevance to more conventional means of socialscience enquiry, this modelling methodology still exists within a preconceived framework of societal interaction, lacking the more external perspective required to develop a fundamental social theory.

Out of Cederman's 3 modelling types, Type 3 models appear to be the most likely to provide a means to draw broader conclusions about the development of societies given that the agents are much less constrained in their interactions than in Type 1 and Type 2 methodologies. However, even models of this type begin with fundamental assumptions: about how these agents will behave, and what sort of interactions might develop. Still we find ourselves striving for a more fundamental perspective, one further removed from an artificially-imposed social framework. We look to systems sociology.

\section{Systems sociology}

As we have seen, the advent of social simulation has proved influential in the social sciences, provoking new questions regarding the origin and nature of society. While Schelling's segregation model demonstrates the potential impact of social simulation, it also illustrates the inherent difficulties involved in generalising the conclusions drawn from a social simulation. More generalised models of society as alluded to by Cederman's Type 3 categorisation may provide a means for investigating aspects of society which elude the empirical data-collector and in turn inform our search for a fundamental social theory, but in order for this to 
occur we need to establish a method of examining society on a broad theoretical scale through simulation.

The well-known social systems theory of Niklas Luhmann provides one example of an attempt to develop an understanding of the foundations for social behaviour. Luhmann classifies social systems as systems of communication which attempt to reduce complexity by presenting only a fraction of the total available information [18].

One of the fundamental issues facing the systems sociology theorist is solving the problem of double contingency, an issue Luhmann describes as central to the development of social order. Put simply, if two entities meet, how do they decide how to behave without a pre-existing social order to govern their actions? How might these entities decide to develop a common means of interaction, and through those interactions develop a shared social history?

As Dittrich, Kron and Banzhaf describe [19], Luhmann described a method for resolving this contingency problem which was far more elemental than previous approaches, relying as it does on 'self-organisation processes in the dimension of time' rather than through more standard social processes. The entities in question would perform initial contingency-reducing actions during an encounter to allow for each to develop an understanding of the expectations of each party in the interaction.

In Luhmann's view, the social order develops as a consequence of these contingency-reducing actions on a large scale. As elements of the developing society develop their expectations about the social expectations of others (described as 'expectation-expectation' by Luhmann), a system of social interaction develops around this mutual social history. This system then produces social institutions which can further influence the development of the social order. These social institutions perform a similar function by reducing the amount of information disseminated amongst the members of a society, essentially providing contingency-reducing services on a much larger scale.

Agent-based models in the context of ALife have certainly proved useful in the examination of other autopoietic systems; however, recent attempts to formalise Luhmann's theories into a usable model, while producing interesting results, have highlighted the inherent difficulties of encapsulating the many disparate elements of Luhmann's theories of social systems into a single model [20].

\section{Evaluation}

Given the outlines of social simulation and systems sociology, this section aims to examine the specific contrasts between these two approaches. By comparing the methods and aims of these methods, we posit that the systems sociology approach presents a promising avenue for developing fundamental social theory through simulation. 


\subsection{Social simulation vs. systems sociology}

As we can see from Luhmann's analysis, while there may indeed be a lack of 'data' inherent to the study of artificial societies, there still exists a theoretical framework for understanding the fundamental mechanisms which drive the creation of a larger social order. While some social simulation researchers may seek to strengthen their models through establishing direct connections with empirically-collected data from social science, the systems sociology perspective could provide a different path to more useful examinations of human society.

The social simulation stream is oriented towards specific elements of social behaviour: simulations of cooperation [21], nationalist insurgency [8] or the spatial patterning of individuals or opinions within a society [13]. Social simulation's stronger links with empirical data may make validation of such models much easier, but further restricts the domain of those models to focus on social problems for which usable data exists. Given the difficulties inherent in collecting social science data, these problems tend to be a subset of those social problems for which models could prove potentially illuminating.

It is the very restriction into particular domains which prevents the social simulation approach from reaching a more general perspective. It is constrained by approaching social phenomena from the top down. These actual top-down approaches are necessarily rooted in the societies they model. In essence, looking for a feature in society and then attempting to reproduce it in a model is not sufficient to develop a fundamental theory.

In contrast, the systems sociology stream abstracts outside of the standard view of society. Luhmann's perspective aims to describe interactions which can lead to the development of social order, in a sense examining the development of human society through an 'outside perspective.' Luhmann essentially moves beyond standard sociology, attempting to describe what occurs prior to the existence of social order, rather than operating within those bounds as with social simulation. By viewing society from its earliest beginnings, prior to the existence of any societally-defined modes of interaction and communication, the systems sociology approach hopes to develop a theoretical understanding of the fundamental behavioural characteristics which lead to the formation of social order. In many ways this approach is reminiscent of the ALife approach to modelling 'life-as-it-could-be'[22]; the systems sociology perspective leads us to examine society-as-it-could-be.

\subsection{The philosophical difficulties of systems sociology}

While the external and abstract perspective offered by systems sociology is appealing given our desire to develop a fundamental social theory, removing ourselves so completely from the conventional empirical perspective carries its own pitfalls. Of course, given that we would not be constructing models with the specificity inherent in the social simulation approach, concerns related to validation and connection with empirically-collected data would not be of any great import. 
However, as Silverman and Bullock describe, developing such broad theories through simulation can carry with it a great deal of philosophical baggage, as in the case of strong ALife [14]. The strong ALife perspective, in which modellers justify their examinations of digital organisms by maintaining that those digital organisms represent a new form of life in the digital substrate, could provoke difficult questions for the systems sociology simulator. Are these artificial societies mere models of societies, or are they bona fide digital cultures in their own right? Are these simulations worthy of attention, or are they merely representational oddities with no direct relation to the development of human society?

Within the systems sociology perspective, however, this modelling approach seeks not to create society but to replicate its beginnings. By allowing agents to construct their own social order, we may be able to observe the micro-level interactions which lead to macro-level social constructs, and do so without a preexisting framework that guides those initial forays into communication. Without question this approach lends itself to difficult philosophical questions, and given the complete separation from conventional empirical social science these questions are even more significant. However, fundamentally this approach brings us full-circle once more to Schelling: we seek models which display a qualitative similarity to our current understanding of social order. If our simplistic computational agents can develop characteristics reminiscent of a society, then we move one step closer to understanding the fundamentals of human society, in the same fashion that Schelling's model provided a novel view of the problem of residential segregation.

\section{Conclusion}

In this paper we have reviewed the state of the art literature in the field of social science modelling. Distinguishing between social simulation and systems sociology has illuminated the shortcomings of social simulation for developing broad theories in social science.

While the production of a fundamental theory of social science is clearly a long way away, the approaches developed through the systems sociology approach should at least be able to play an instructive or forming role in the development of social theory. Computer models that are at first very complex are still mathematical constructs and may well be simplified to explicit mathematical models which form competent explanations [23]. By taking this bottom-up approach of building new societies, while maintaining a top-down motivation, new directions can emerge in future modelling endeavours.

Acknowledgments: Thanks to Takashi Ikegami and David Hogg.

\section{References}

1. Darwin, C.: The Origin of Species by Means of Natural Selection. John Murray, London (1859) 
2. Schelling, T.C.: Dynamic models of segregation. Journal of Mathematical Sociology 1 (1971) 143-186

3. Schelling, T.: Micromotives and macrobehavior. W. W. Norton (1978)

4. Sander, R., Schreiber, D., Doherty, J.: Empirically testing a computational model: The example of housing segregation. In Sallach, D., Wolsko, T., eds.: Proceedings of the Workshop on Simulation of Social Agents: Architectures and Institutions. (2000) 108-115

5. Clark, W.A.V.: Residential preferences and neighborhood racial segregation: A test of the schelling segregation model. Demography 28 (1991) 1-19

6. Epstein, J.M., Axtell, R.L.: Growing Artificial Societies: Social Science from the Bottom Up. MIT Press, Cambridge, MA (1996)

7. Arthur, W.B.: Competing technologies, increasing returns, and lock-in by historical events. Journal of Economics 116 (1989) 99

8. Cederman, L.E.: Emergent actors in world politics: how states and nations develop and dissolve. Princeton University Press (1997)

9. Cederman, L.E.: Agent-based modeling in political science. The Political Methodologist 10 (2001) 16-22

10. Axelrod, R.: The Evolution of Cooperation. Basic Books, New York (1984)

11. Cederman, L.E., Girardin, L.: Beyond fractionalization: Mapping ethnicity onto nationalist insurgencies. Paper read at Disaggregating the Study of Civil War and Transnational Violence Conference, at San Diego, CA (2005)

12. Cederman, L.E.: Articulating the Geo-Cultural Logic of Nationalist Insurgency. In Order, Conflict, and Violence. Cambridge University Press (2006)

13. Lustick, I.: Defining violence: a plausibility probe using agent-based modeling. Paper prepared for LiCEP, Princeton University, May 12-14 (2006)

14. Silverman, E., Bullock, S.: Empiricism in artificial life. In Pollack, J., Bedau, M., Husbands, P., Ikegami, T., Watson, R.A., eds.: Artificial Life IX: Proceedings of the Ninth International Conference on the Simulation and Synthesis of Living Systems, MIT PRess (2004) 534-539

15. Klüver, J., Stoica, C., Schmidt, J.: Formal models, social theory and computer simulations: Some methodical reflections. Journal of Artificial Societies and Social Simulation 6(2)8 (2003) [online] http://jasss.soc.surrey.ac.uk/6/2/8.html.

16. Sylvan, D.: 'modeling the rise and fall of states', review of emergent actors in world politics by lars-erik cederman. Mershon International Studies Review 42 (1998) 377-379

17. Weidmann, N.B., Girardin, L.: GROWLab: A Toolbox for Social Simulation. Paper presented at the First World Congress on Social Simulation, August 21-25, 2006 in Kyoto, Japan. (2006)

18. Luhmann, N.: Social Systems. Stanford University Press (1995)

19. Dittrich, P., Kron, T., Banzhaf, W.: On the scalability of social order: Modeling the problem of double and multi contingency following luhmann. Journal of Artificial Societies and Social Simulation 6(1)3 (2003) [online] http://jasss.soc.surrey . ac.uk/6/1/3.html.

20. Fleischmann, A.: A model for a simple luhmann economy. Journal of Artificial Societies and Social Simulation 8(2)4 (2005) [online] http://jasss.soc.surrey . ac.uk/8/2/4.html.

21. Axelrod, R.: The Complexity of Cooperation. Princeton University Press (1997)

22. Langton, C.: Artificial life. In Langton, C., ed.: Artificial Life. Addison-Wesley (1989) $1-47$

23. Bryden, J., Noble, J.: Computational modelling, explicit mathematical treatments, and scientific explanation. Artificial Life X (2006) 520-526 